\title{
Subject Index, Vol. 68, 1995
}

This index does not contain items of the reports of the Chromosome Mapping Workshops 3, 8 , and 16 which are published in this volume.

Animals

cattle 102

chimpanzee 25

Chinese hamster 95

lesser apes 74

Macropus eugeníi (tammar wallaby) 64

mouse $19,33,54,185,197$

Old World monkeys 74

rat 107,239

sheep 85, 102

Sparus aurata (gilthead seabream fish) 3

Syrian hamster 71

Banded chromosome analysis

gilthead seabream(Ag, C, CM A3, DAPI) 3

human(CMA3) 192

human(DAPI) 25, 33, 49, 61, 122, 231

human $(\mathrm{G}) 25,67,79,91$

human(Q) 52,233

human(R) 45, 112,235

mouse(C) 54

primates(Ag, DAPI, G) 74

sheep $(\mathrm{G}, \mathrm{R})$ 85,102

sheep (Q) 102

Syrian hamster(DAPI) 71

Chromosome aberration

age-associated micronuclei 11

double minutes (DMs) 54

interstitial deletion of 2p21 17

NOR deletion in fish 3

trisomy 21203

unstable HSR 33 Chromosome mapping workshops

chromosome 16 report 165

chromosome 3 report 125

chromosome 8 report 147 Comparative gene mapping

cattle 102

mouse 19,185

primate 25 
rat 107,239

sheep 102

Syrian hamster 71

Fluorescent in situ hybridization (FISH) age-associated micronuclei formation 11 chromosomal painting of primates 74 chromosome painting in mouse 197 detection of aneuploidy at interphase 203

gene mapping $8,19,45,52,61,67,71,79$,

$91,112,119,122,185,192,207,222$,

226,231,233,235,239 instant PRINS 57 kinetochore in DMs 54 PRINS of human telomeres

115 proposed nomenclature system for painted

chromosomes 211 spontaneous amplification of interstitial

telomeric bands 95 subchromosomal painting libraries (SCPLs)

25 unstable HSR 33

Gene mapping human

11 probes in $5 \mathrm{q} 2 \rightarrow \mathrm{q} 3207$

300 regions defined by SCPLs 25

434 cosmid markers on chromosome 2112

5SrRNA 82

AQP2, MIP 45

ARF2, NCK 91

Chromosome 3- $\Lambda^{1 / 8} / 1$-linking clones 91

D16S444E 49

FBLN1 192

GASl,NBCCS 119

LCAT, D16S124 194

MC5R 79

MCM2 226

MDC 39

microclones in 2p2 $3 \rightarrow \mathrm{p} 2117$

NFATC1, NFATC2 (formerly NFATc, NFATp) 185

OTF1 231

SCN5A 67

SFTP3 8

TCF12 235

TPM3 122

U2AFBPL 19

UND 233

WEEl, ST5, rhombotin 222

$\mathrm{XDH}$ 52,61 mouse

Nfatcl, Nfatd (formerly Nfatc, Nfatp) 185

U2afipL 19 rat

8 microsatellite markers 107

Cstrp 239

sheep

CA2, CALB1, CRH, CYP11B1, IL7 102

Syrian hamster Tp53 71 
Genomic structure

alternative splicing of MCD 39 exon-intron organization of MCD 39

Human disorders

Beckwith-Wiedemann syndrome 222

nevoid basal cell carcinoma syndrome (NBCCS) 119

xanthinuria 52, 61 Hybrids

chimpanzee-hamster 25

human-hamster 25, 67, 185

human-mouse 49, 235

human-rat 235

mouse-hamster 185

mouse-rat $107,185,239$

sheep-hamster 102

subchromosomal painting libraries generated from 25

Ideogram sheep 85

Karyotype sheep 85

Linkage rat 107 tammar wallaby 64

Microdissection CM-PCR 67 library of 2p2 $3 \rightarrow$ p21 17

Physical mapping 1lp15.3 222 16q22.1 194

Techniques

chromosome painting in mouse 197 instant PRINS for clinical diagnosis 57 PRINS to stain human telomeres 115 subchromosomal painting libraries 25

244

Cytogenet Cell Genet, Vol. 68,1995 